\title{
HMGA2 protein expression correlates with lymph node metastasis and increased tumor grade in pancreatic ductal adenocarcinoma
}

\author{
Alexandra C Hristov ${ }^{1}$, Leslie Cope ${ }^{2,3}$, Marcelo Delos Reyes ${ }^{1}$, Mansher Singh ${ }^{1}$, \\ Christine Iacobuzio-Donahue ${ }^{1,2}$, Anirban Maitra ${ }^{1,2}$ and LMS $\operatorname{Resar}^{2,4,5}$ \\ ${ }^{1}$ Department of Pathology, The Johns Hopkins University School of Medicine, Baltimore, MD, USA; \\ ${ }^{2}$ Department of Oncology, The Johns Hopkins University School of Medicine, Baltimore, MD, USA; \\ ${ }^{3}$ Department of Biostatistics, The Johns Hopkins University School of Medicine, Baltimore, MD, USA; \\ ${ }^{4}$ Department of Medicine, The Johns Hopkins University School of Medicine, Baltimore, MD, USA and \\ ${ }^{5}$ Department of Pediatrics, The Johns Hopkins University School of Medicine, Baltimore, MD, USA
}

\begin{abstract}
Pancreatic ductal adenocarcinoma is a highly aggressive, lethal human malignancy that continues to elude successful treatment. Although most patients present with metastatic disease, the molecular pathways that underlie tumor progression and metastases are poorly understood. The high mobility group A2 (HMGA2) protein is an architectural transcription factor that has recently been implicated in the development and progression of malignant tumors. Here, we examined HMGA2 gene expression in pancreatic ductal adenocarcinoma to determine if it could be a marker for more advanced disease. By real time quantitative RT-PCR, we showed a marked increase in HMGA2 mRNA in two of three cultured pancreatic ductal adenocarcinoma cell lines compared to normal pancreatic tissue. Using tissue microarrays generated from 124 pancreatic ductal adenocarcinoma cases, we also assessed HMGA2 protein levels by immunohistochemical analysis. We found that HMGA2 nuclear immunoreactivity correlates positively with lymph node metastases and high tumor grade. Our results support a role for HMGA2 in the progression of pancreatic ductal adenocarcinoma and suggest that it could be a useful biomarker and rational therapeutic target in more advanced disease.
\end{abstract}

Modern Pathology (2009) 22, 43-49; doi:10.1038/modpathol.2008.140; published online 29 August 2008

Keywords: HMGA2; pancreatic ductal adenocarcinoma; oncogene; immunoreactivity

Pancreatic ductal adenocarcinoma is a common, highly lethal malignancy accounting for over 30000 deaths in America and 160000 deaths worldwide each year. Unfortunately, current therapies, including surgery, chemotherapy, and radiation therapy, are largely ineffective and death rates closely parallel incidence rates. ${ }^{1,2}$ Most patients with pancreatic ductal adenocarcinoma present with locally advanced or metastatic disease that renders them inoperable or limits the effectiveness of therapy. Moreover, even most patients with small lesions ultimately succumb to advanced disease with distant metastases, despite adjuvant therapy.

Correspondence: Dr LMS Resar, MD, Department of Medicine or AC Hristov, MD, Department of Pathology, The Johns Hopkins University School of Medicine, Ross Research Building, Room 1025, 720 Rutland Avenue, Baltimore, MD 21205, USA.

E-mails: lresar@jhmi.edu or acappie1@jhmi.edu

Received 30 June 2008; revised and accepted 01 August 2008; published online 29 August 2008
These findings emphasize the importance of understanding the molecular events that lead to tumor progression. Although molecular events that contribute to the early development of pancreatic ductal adenocarcinoma have been elucidated, those that orchestrate tumor progression and metastasis are poorly understood.

The HMGA genes encode a family of the high mobility group (HMG) proteins, which were discovered over 25 years ago as abundant, nonhistone chromatin binding proteins and named for their rapid electrophoretic mobility in polyacrylamide gels. ${ }^{3-5}$ HMGA1a and HMGA1b protein isoforms result from alternative splicing of the HMGA1 mRNA, ${ }^{3-5}$ whereas HMGA2 is encoded by the separate, but related gene, HMGA2. ${ }^{6,7}$ All HMGA proteins contain three AT hook DNA-binding motifs that mediate binding to AT-rich regions in the minor groove of chromosomal DNA. ${ }^{8,9}$ Once bound to DNA, the HMGA proteins recruit additional transcription factors, and in concert with these factors, 
alter gene expression. ${ }^{10-13}$ Although they do not regulate transcription alone, through protein-protein, and protein-DNA interactions, they form a higher order transcriptional complex or "enhanceosome' with additional proteins that appears to be essential for the efficient expression of specific target genes. Because these proteins alter DNA structure, they have been called architectural transcription factors. ${ }^{10-13}$ Normally, HMGA genes are expressed at high levels during embryonic development, with only low levels in most adult, differentiated tissues. ${ }^{14-16}$ Like many transcriptional regulators, HMGA proteins participate in diverse cellular functions, including cell cycle regulation, differentiation, senescence, and neoplastic transformation.

Although previous studies suggest that HMGA1 functions as an oncogene with overexpression in diverse, human cancers, the role of HMGA2 in cancer is not well understood. The HMGA2 gene is located on chromosome 12q13-15 and encodes a 109 amino-acid protein. Rearrangements involving HMGA2 are a prominent feature of benign, mesenchymal tumors, ${ }^{10-12,16-19}$ although emerging evidence implicates HMGA2 in the pathogenesis of malignant tumors. Recent studies report increased expression of HMGA2 in epithelial tumors, including lung ${ }^{17,20}$ and pancreatic tumors, ${ }^{21}$ oral squamous cell carcinomas ${ }^{22}$ and a subset of breast cancers. ${ }^{23}$ Increased expression of HMGA2 was also reported in the peripheral blood of patients with chronic myelogenous leukemia. ${ }^{20}$ Moreover, translocations involving HMGA2 associated with increased expression have been discovered in myeloproliferative disorders. ${ }^{20,24-26}$ Taken together, these studies suggest that HMGA2 may contribute to neoplastic transformation in the malignancies in which it is overexpressed.

Here, we demonstrate that HMGA2 is overexpressed in more advanced pancreatic ductal adenocarcinoma. Although further studies are needed, our findings suggest that HMGA2 could contribute to disease progression and may represent a potential biomarker and rational therapeutic target in pancreatic ductal adenocarcinoma.

\section{Materials and methods}

\section{Cultured Human Pancreatic Ductal Adenocarcinoma Cells and Normal Human Pancreas}

The human pancreatic ductal adenocarcinoma cell lines MIA PaCa-2 (CRL-1420) and PL-1 (CRL-2554, Panc 02.13) were obtained from the American Type Culture Collection and were grown as recommended. MIA PaCa-2 cells are derived from a 65-year-old Caucasian man with an undifferentiated pancreatic carcinoma that involved the pancreatic body and tail with fixation to the periaortic soft tissues. ${ }^{27}$ PL-1 cells were generated from a primary pancreatic ductal adenocarcinoma in a 64-year-old
Caucasian woman that extended to involve the duodenum, but did not metastasize to lymph nodes. ${ }^{28}$ The human pancreatic cancer cell line XPA-3 (PX154) was established from a primary pancreatic ductal adenocarcinoma in a patient with known lymph node metastases. ${ }^{29}$ Normal human pancreas was obtained from a normal 35-year-old man (Becton Dickinson, San Jose, CA, USA).

\section{Quantitative RT-PCR of Cultured Human Pancreatic Ductal Adenocarcinoma Cells and Normal Human Pancreas}

Quantitative RT-PCR was performed by Taqman chemistry as we previously described ${ }^{30}$ except that we used the HMGA2-specific primers and probe as follows: $5^{\prime}$-AAAGCAGCTCAAAAGAAAGCA-3' (5' primer), $5^{\prime}$-TGTTGTGGCCATTTCCTAGGT- ${ }^{\prime}$ ( $3^{\prime}$ primer) and the HMGA2-specific probe $5^{\prime}$-CACT GGAGAAAAACGGCCAAG-3'.

\section{Tissue Microarrays}

Tissue microarray blocks were created using 2-mm cores taken from 124 archival, routinely processed, paraffin-embedded, pancreatic ductal adenocarcinoma specimens from the surgical pathology archives of The Johns Hopkins Medical Institutions, Baltimore, MD, USA. The specimens had been accessioned between 2000 and 2003 and were selected solely based on availability of pancreatic ductal adenocarcinoma in the block. Each case was represented by four cores on the tissue microarrays, including two cores taken from tumor in the pancreatic parenchyma and two cores taken from tumor in the peripancreatic soft tissue and lymph nodes. Cores from routinely processed, paraffin-embedded normal tissues from the Johns Hopkins Medical Institution archives were also represented on the arrays, including bladder, brain, breast, colon, gallbladder, kidney, liver, lung, pancreas, salivary gland, skin, small intestines, spleen, stomach, testis, and tonsil tissue. Pathologic diagnosis, grade and stage were previously determined for each case by attending pathologists of The Johns Hopkins Hospital Department of Surgical Pathology and this information was retrieved from the medical record. Patient survival information was also obtained from the medical record.

\section{Immunohistochemistry}

The tissue microarrays were sectioned at $4 \mu \mathrm{m}$ intervals, deparaffinized in xylene and rehydrated with graded alcohols. Sections were then heated in antigen retrieval solution (Dako, Carpinteria, CA, USA) and were immunohistochemically stained for HMGA2 protein following manufacturer directions (HMGA2, $0.004 \mathrm{mg} / \mathrm{ml}$, BioCheck Inc., Foster City, 
CA, USA). We showed previously that this antibody is specific for HMGA2. ${ }^{31}$ At the dilutions used in this study, there was no significant cross-reactivity with HMGA1 (unpublished results) using an antibody shown to be specific for HMGA1 (Santa Cruz, $\mathrm{N}-19$ ). Specific nuclear immunoreactivity was considered positive staining and was scored from 5 to 300 based on the product of staining intensity (weak $=1$, moderate $=2$, strong $=3$ ) and staining extent (percent of positive cells, 5-100\%). The highest nuclear immunoreactivity score per case was used for subsequent analyses. The tissue microarrays were analysed microscopically in a blinded fashion by a single investigator (AH).

\section{Statistical Analysis}

Results are expressed as the mean \pm s.e. The Student's $t$-test was used to compare HMGA2 mRNA levels from pancreatic ductal adenocarcinoma cells and normal tissue. To determine if HMGA2 nuclear immunoreactivity correlated with more advanced disease, the following clinical parameters were investigated: overall survival, tumor grade, and lymph node metastases. Correlation between staining extent and intensity was determined by Spearman's correlation coefficient. HMGA2 protein levels were compared to tumor grade and lymph node involvement using the Student's $t$-test. A $\chi^{2}$-test was used to evaluate the relationship between tumor grade and survival and the relationship between HMGA2 immunoreactivity and survival.

\section{Results}

\section{Patient Characteristics}

Mean patient age was 67.5 years (range 32-90 years); 60 patients were female and 64 patients were male. Table 1 shows the available stage, grade and survival information for all patients. Tumor grade was available for 122 of the 124 patients and survival information was available for 119 of 124 patients.

Table 1 Distribution of cases by stage, lymph node involvement, grade and mean survival

\begin{tabular}{|c|c|c|c|c|c|c|c|}
\hline \multirow[b]{2}{*}{ Stage } & \multirow[b]{2}{*}{$\mathrm{n}$} & \multirow[b]{2}{*}{$N 1^{\mathrm{a}}$} & \multicolumn{4}{|c|}{ Grade } & \multirow{2}{*}{$\begin{array}{l}\text { Survival } \\
\text { (months) }\end{array}$} \\
\hline & & & 1 & 2 & 3 & Unknown & \\
\hline $\mathrm{Ia} / \mathrm{b}$ & 3 & 0 & 0 & 2 & 1 & & 31.0 \\
\hline IIa & 18 & 0 & 2 & 10 & 6 & & 17.4 \\
\hline IIb & 100 & 100 & 2 & 53 & 43 & 2 & 17.3 \\
\hline III & 3 & 2 & 0 & 1 & 2 & & 13.3 \\
\hline
\end{tabular}

${ }^{\mathrm{a}} \mathrm{N} 1$ indicates lymph node involvement.
HMGA2 Overexpression in Pancreatic Ductal Adenocarcinoma Cells

To determine if HMGA2 is overexpressed in pancreatic ductal adenocarcinoma, we measured HMGA2 mRNA levels by quantitative RT-PCR in cultured pancreatic ductal adenocarcinoma cells. Strikingly, we observed markedly increased levels of HMGA2 mRNA in the cultured cells derived from two of three pancreatic ductal adenocarcinoma cell lines (Figure 1). Specifically, HMGA2 mRNA was increased over 800-fold in both the XPA-3 $(P=0.0016$ by Student's $t$-test) and PL-1 cells $(P=0.0004$ by Student's $t$-test). Both of these cell lines were generated from pancreatic ductal adenocarcinoma that had extended outside of the pancreas. PL-1 cells originate from a primary tumor that extended to the duodenum and XPA-3 cells were generated from a primary tumor that metastasized to the lymph nodes. In contrast, MIA PaCa-2 cells showed levels similar to the control tissue. These cells were derived from an undifferentiated pancreatic tumor that involved the pancreas with fixation to periaortic soft tissue.

\section{HMGA2 Immunoreactivity in Pancreatic Ductal Adenocarcinoma}

Given the significant overexpression of $H M G A 2$ in a subset of pancreatic ductal adenocarcinoma cell lines, we sought to determine if HMGA2 is over-

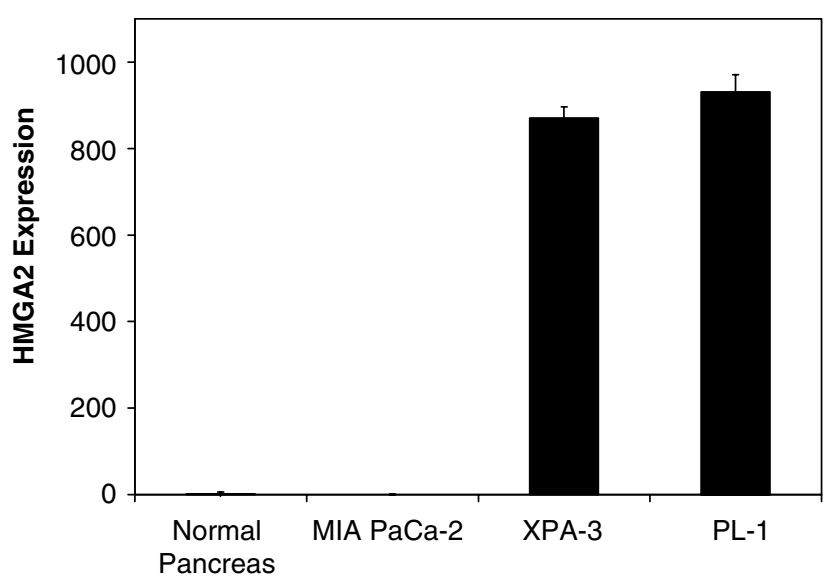

Figure 1 HMGA2 mRNA expression in human pancreatic ductal adenocarcinoma cell lines. The HMGA2 mRNA levels were measured by quantitative RT-PCR and normalized to human phosphoprotein as a loading control. Normal pancreatic tissue from a healthy 35-year-old donor was used as a control and assigned a value of 1 . HMGA2 mRNA levels are significantly elevated above control in two of three pancreatic ductal adenocarcinoma cell lines (871-fold $\pm 26, P=0.0016$ for the XPA-3 cells and 931-fold $\pm 40, P=0.0004$ for the PL- 1 cells, both by Student's $t$-test). MIA PaCa-2 cells express amounts of HMGA2 similar to normal pancreatic tissue $(0.29 \pm 0.1$ for MIA PaCa-2 cells vs $1 \pm 5$ for normal human pancreas). Two independent experiments were performed in triplicate; results represent the mean \pm s.e. from independent experiments. 
expressed at the level of the protein in primary pancreatic ductal adenocarcinoma cases compared to normal tissue or pancreatic intraepithelial neoplasia (PanIN). PanINs are well-described precursor lesions that are thought to progress through a series of morphologic and molecular events leading to invasive pancreatic cancer. ${ }^{32}$ To this end, we investigated HMGA2 protein levels by immunohistochemical analysis in 124 pancreatic ductal adenocarcinoma cases represented on a tissue microarray. We identified HMGA2 nuclear immunoreactivity in 55 of 124 (44.4\%) pancreatic ductal adenocarcinomas, with scores ranging from 5 to $300 \%$. Notably, the strength of staining and extent of staining were highly correlated ( $r=0.95$ by Spearman's correlation coefficient). Of positive cases, $22(40 \%)$ displayed limited immunoreactivity (score <50), 18 (33\%) displayed moderate immunoreactivity (score 50$150)$ and $15(27 \%)$ displayed strong immunoreactivity (score >150; Figure 2). There was no staining for HMGA2 in the PanIN precursor lesions (grades 1-3) that were present in six pancreatic ductal adenocarcinoma cases. Moreover, there was no staining observed in the normal tissues, consistent with prior reports that this protein is not expressed in terminally differentiated tissues. ${ }^{15,16,22}$

\section{HMGA2 Expression Correlates with Lymph Node Metastases and Poor Histopathologic Differentiation}

Because we found elevated HMGA2 mRNA and protein levels in pancreatic ductal adenocarcinoma cells, but not in normal pancreatic tissue, we hypothesized that HMGA2 expression could be a biomarker for more advanced disease. We therefore determined if HMGA2 protein levels correlate with clinical parameters indicative of disease progression, including lymph node involvement, poor differentiation (high grade) or survival. We found significantly higher HMGA2 immunoreactivity scores in pancreatic ductal adenocarcinoma samples
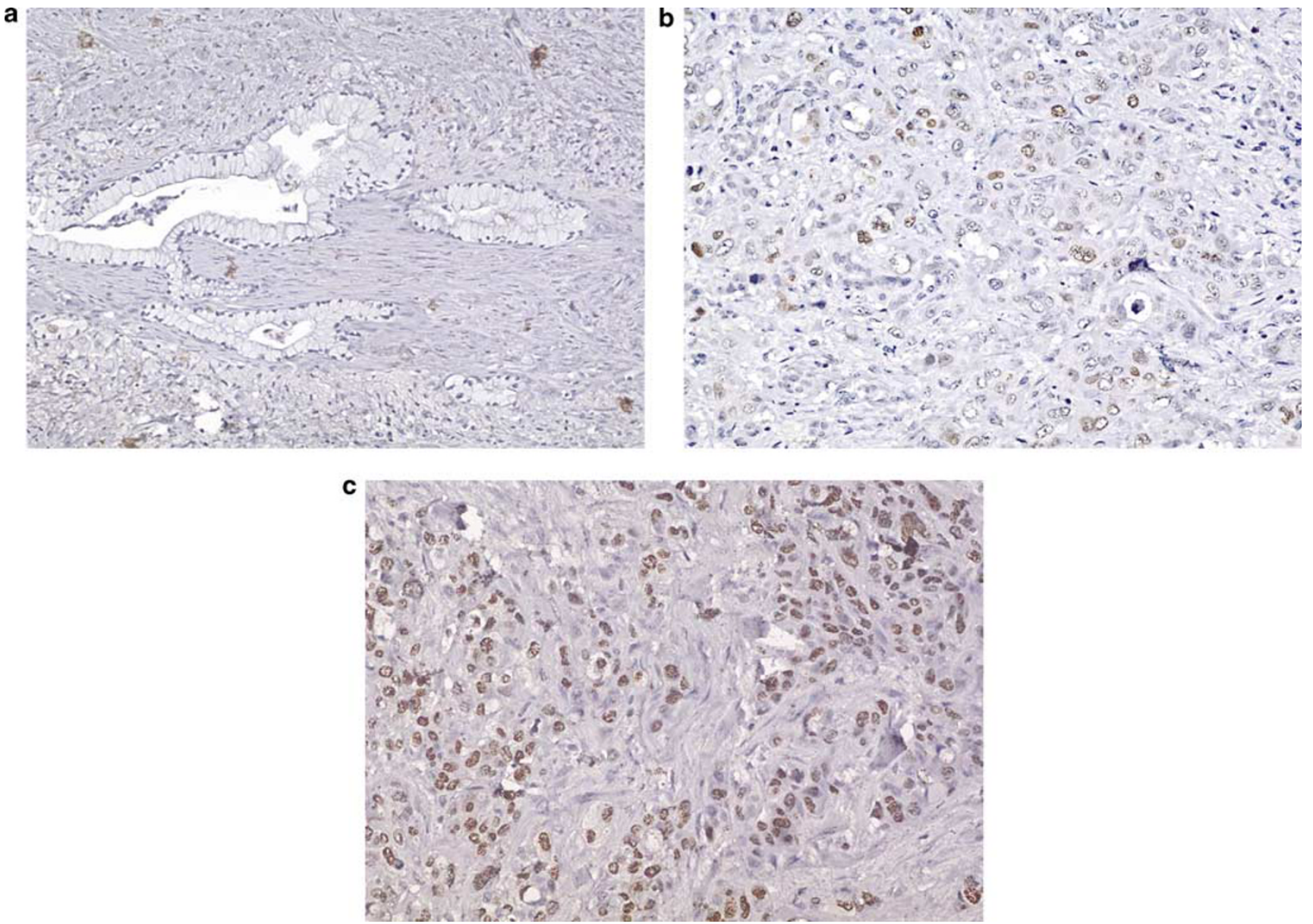

Figure 2 Immunohistochemical staining for HMGA2 protein in pancreatic ductal adenocarcinoma on the tissue microarray (HMGA2 antibody, Gill hematoxylin counterstain). (a) Absent HMGA2 staining in a well-differentiated pancreatic ductal adenocarcinoma. The infiltrating glands do not display nuclear immunoreactivity (magnification $\times 40$, score 0 ). (b) Moderate HMGA2 staining in a moderately differentiated pancreatic ductal adenocarcinoma. The infiltrating, crowded glands display light background nuclear immunoreactivity and intermittent moderate-to-strong nuclear reactivity (magnification $\times 64$, score 50-150). (c) Strong HMGA2 staining in a poorly differentiated pancreatic ductal adenocarcinoma. The infiltrating cells with enlarged nuclei and minimal gland formation displayed marked nuclear immunoreactivity (magnification $\times 64$, score $>150$ ). 
with lymph node metastases compared to those without lymph node involvement $(51.5 \pm 8.4 \mathrm{VS}$ $19.4 \pm 8.8$ (mean \pm s.e.); $P=0.011$ by Student's t-test; Figure 3a). Moreover, HMGA2 immunoreactivity was present in only 6 of 22 cases $(27 \%)$ with negative lymph node involvement, but 49 of 102 $(48 \%)$ with lymph node involvement. We also observed significantly higher HMGA2 immunoreactivity in poorly differentiated pancreatic ductal adenocarcinoma tumor samples compared to well to moderately differentiated tumor samples $(62.7 \pm 13.9$ vs $32.9 \pm 6.9 ; \quad P=0.042$ by Student's $t$-test; Figure $3 \mathrm{~b})$. Although we did not observe a relationship between survival and HMGA2 score, there was a strong relationship between grade and survival ( $P=0.0002$ by $\chi^{2}$-analysis). Taken together, our results indicate that HMGA2 could serve as a useful biomarker for tumor progression in pancreatic ductal adenocarcinoma.

\section{Discussion}

Pancreatic ductal adenocarcinoma is a devastating disease with a nearly identical incidence and death rate. $^{1,2}$ Moreover, it is the fourth most common cause of cancer-related mortality in the United States. The abysmal epidemiology of pancreatic ductal adenocarcinoma reflects the ineffectiveness of current therapies and the urgent need for further research to identify novel therapeutic strategies. Despite the high incidence of pancreatic ductal adenocarcinoma, there is limited knowledge regarding the late molecular events that enable a localized tumor to transition to metastatic disease. The K-RAS oncogene is frequently activated by point mutations in sporadic pancreatic cancer, although these mutations typically occur early in carcinogenesis at the earliest (PanIN-1) precursor lesion stage. ${ }^{32}$ Loss of function of p16 (INK4A), either by deletion, methylation, or mutation, is another common genetic mutation that occurs in slightly more advanced precursor lesions (PanIN-2). Loss of p16 occurs at a frequency of 80-95\% in PanIN-2 lesions that arise in association with sporadic pancreatic cancer. ${ }^{33,34}$ Inactivating mutations in the p53 tumor suppressor occur at a frequency of about 50-75\% in the PanIN-3 precursor lesions arising in association with sporadic pancreatic cancer. ${ }^{34}$ Although these studies have uncovered molecular events that correspond to early progression in precursor lesions, this knowledge has not translated to improved therapy or outcome for patients with pancreatic ductal adenocarcinoma. The identification of molecular pathways that mediate later events in tumor progression may provide novel therapeutic targets and ultimately lead to improved survival.

Recent studies have implicated HMGA2 in the development and progression of human carcinomas. ${ }^{16,18,24-26}$ For example, HMGA2 protein expression localizes to the invasive tumor front
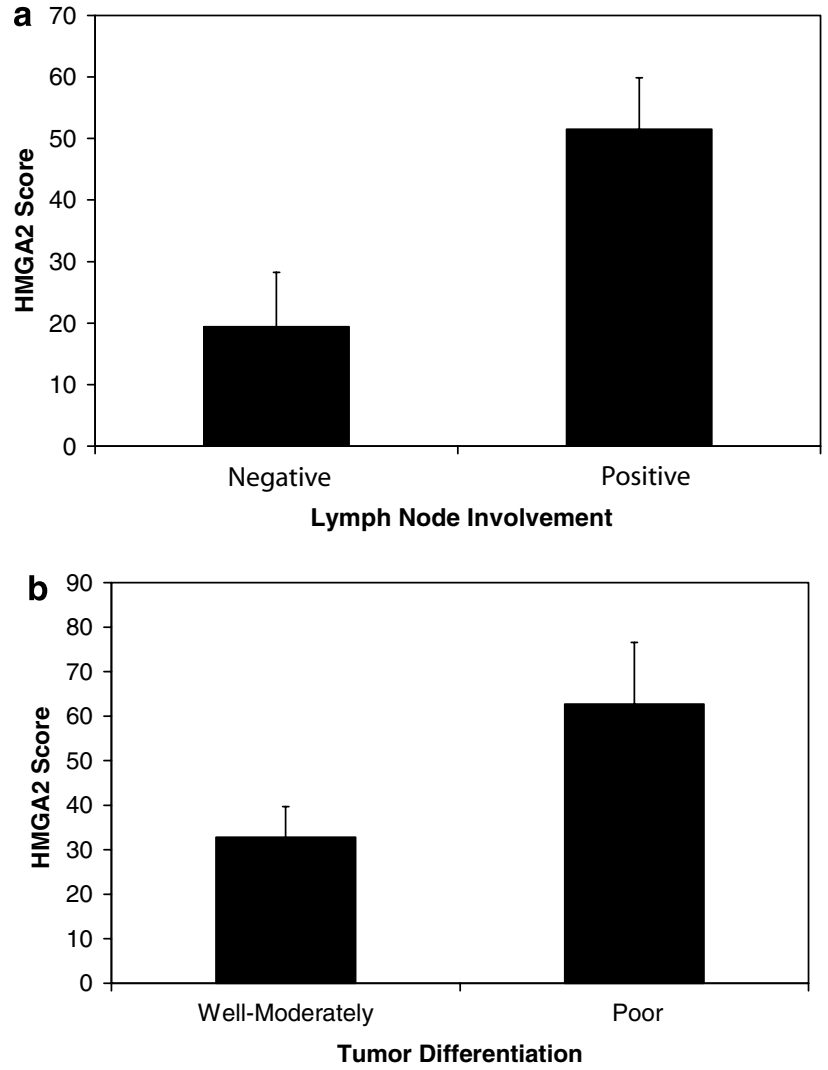

Figure 3 HMGA2 immunoreactivity correlates with lymph node involvement and tumor differentiation in pancreatic ductal adenocarcinoma. (a) Graphical representation of mean HMGA2 immunoreactivity score in pancreatic ductal adenocarcinoma cases with lymph node involvement compared to those without lymph node involvement. Note that the HMGA2 score is significantly higher in cases with lymph node involvement compared to those without $(P=0.011$ by Student's $t$-test $)$. Bars \pm s.e. are shown. (b) Graphical representation of mean HMGA2 immunoreactivity in pancreatic ductal adenocarcinoma cases that are well to moderately differentiated compared to poorly differentiated. Note the significantly higher HMGA2 immunoreactivity score in the poorly differentiated cases $(P=0.042$ by Student's $t$-test). Bars \pm s.e. are shown.

histopathologically and correlates with lymph node metastases and decreased survival in oral squamous cell carcinoma. ${ }^{22}$ A previous study of primary lung adenocarcinomas also showed that high levels of HMGA2 nuclear staining correlate with poor survival. ${ }^{19}$ In addition, a small study of invasive ductal breast cancers showed evidence for HMGA2 gene expression only in the higher grade lesions. ${ }^{19}$ Moreover, HMGA2 appears to be a key factor involved in the epithelial-mesenchymal transition, which occurs during tumor invasion and metastasis in carcinomas. ${ }^{35} \mathrm{~A}$ recent study showed that HMGA2 is a direct transcriptional target of transforming growth factor- $\beta$, an epithelial-mesenchymal transition initiator. Following upregulation by transforming growth factor- $\beta$, HMGA2 activates expression of key transcription factors involved in the epithelialmesenchymal transition including snail, slug, and twist. ${ }^{35}$ Its function in the epithelial-mesenchymal 
transition and high expression in more advanced disease suggest that HMGA2 may be an important contributor to tumor progression.

Here, we demonstrate a significant, positive correlation between HMGA2 protein levels and more advanced disease. Specifically, a high immunoreactivity score correlates with both advanced tumor grade and lymph node metastases. We did not observe a correlation between HMGA2 score and survival, possibly because of limited patient survival, as most patients with pancreatic ductal adenocarcinoma expire quickly from their disease. A prior study showed evidence for HMGA2 gene expression by RT-PCR in 17 cases of pancreatic ductal adenocarcinoma, although the RT-PCR was not quantitative and expression was also noted in normal pancreas and chronic pancreatitis by their approach. ${ }^{21}$ Immunoreactivity was observed in $27 / 27$ pancreatic ductal adenocarcinoma cases; however, tumor grade and clinical outcomes were not included in this analysis. Our study is the first to report a correlation between HMGA2 immunoreactivity and poor differentiation or lymph node metastases in pancreatic ductal adenocarcinoma. Of note, the prior study reported abundant HMGA2 immunoreactivity in non-neoplastic islet cells and focal immunoreactivity in duct epithelial cells, findings not observed in this study and possibly reflecting a difference in sensitivity and specificity between immunohistochemical assays. Although HMGA2 expression has been reported in pancreatic ductal epithelium in the mouse embryo up to day 17.5 postconception, it was not found in pancreatic islets. $^{14}$ Interestingly, HMGA2 was not overexpressed in MIA PaCa-2 cells, which are derived from an undifferentiated pancreatic carcinoma. Our findings may reflect a different molecular pathway for this tumor compared to more conventional pancreatic ductal adenocarcinoma.

In summary, we found that HMGA2 gene expression is increased in pancreatic ductal adenocarcinoma cell lines and HMGA2 immunoreactivity correlates with poor tumor differentiation and lymph node metastases. Our findings, together with emerging evidence indicating a role for HMGA2 in tumor progression, suggest that HMGA2 could serve as a useful biomarker and potential therapeutic target in pancreatic ductal adenocarcinoma and other cancers in which it is overexpressed.

\section{Disclosure/Conflict of interest}

No relevant financial or other disclosures or conflicts of interest.

\section{References}

1 Jemal A, Siegel R, Ward E, et al. Cancer statistics, 2007. CA Cancer J Clin 2007;57:43-66.
2 Yeo TP, Hruban RH, Leach SD, et al. Pancreatic cancer. Curr Probl Cancer 2002;26:176-275.

3 Johnson KR, Cook SA, Davisson MT. Chromosomal localization of the murine gene and two related sequences encoding high-mobility-group I and $\mathrm{Y}$ proteins. Genomics 1992;12:503-509.

4 Johnson KR, Lehn DA, Elton TS, et al. Complete murine cDNA sequence, genomic structure, and tissue expression of the high mobility group protein HMGI(Y). J Biol Chem 1988;263:18338-18342.

5 Lund T, Holtlund J, Fredriksen M, et al. On the presence of two new high mobility group-like proteins in HeLa S3 cells. FEBS Lett 1983;152:163-167.

6 Giancotti V, Bandiera A, Buratti E, et al. Comparison of multiple forms of the high mobility group I proteins in rodent and human cells. Identification of the human high mobility group I-C protein. Eur J Biochem 1991;198:211-216.

7 Ishwad CS, Shriver MD, Lassige DM, et al. The high mobility group I-C gene (HMGI-C): polymorphism and genetic localization. Hum Genet 1997;99:103-105.

8 Bustin M. Regulation of DNA-dependent activities by the functional motifs of the high-mobility-group chromosomal proteins. Mol Cell Biol 1999;19: 5237-5246.

9 Bustin M. Revised nomenclature for high mobility group (HMG) chromosomal proteins. Trends Biochem Sci 2001;26:152-153.

10 Fusco A, Fedele M. Roles of HMGA proteins in cancer. Nat Rev Cancer 2007;7:899-910.

11 Reeves R. Molecular biology of HMGA proteins: hubs of nuclear function. Gene 2001;277:63-81.

12 Sgarra R, Rustighi A, Tessari MA, et al. Nuclear phosphoproteins HMGA and their relationship with chromatin structure and cancer. FEBS Lett 2004;574: 1-8.

13 Wolffe AP. Architectural transcription factors. Science 1994;264:1100-1101.

14 Hirning-Folz U, Wilda M, Rippe V, et al. The expression pattern of the Hmgic gene during development. Genes Chromosomes Cancer 1998;23:350-357.

15 Li O, Li J, Droge P. DNA architectural factor and protooncogene HMGA2 regulates key developmental genes in pluripotent human embryonic stem cells. FEBS Lett 2007;581:3533-3537.

16 Rogalla P, Drechsler K, Frey G, et al. HMGI-C expression patterns in human tissues. Implications for the genesis of frequent mesenchymal tumors. Am J Pathol 1996;149:775-779.

17 Sarhadi VK, Wikman H, Salmenkivi K, et al. Increased expression of high mobility group A proteins in lung cancer. J Pathol 2006;209:206-212.

18 Schoenmakers EF, Wanschura S, Mols R, et al. Recurrent rearrangements in the high mobility group protein gene, HMGI-C, in benign mesenchymal tumours. Nat Genet 1995;10:436-444.

19 Young AR, Narita M. Oncogenic HMGA2: short or small? Genes Dev 2007;21:1005-1009.

20 Meyer B, Loeschke S, Schultze A, et al. HMGA2 overexpression in non-small cell lung cancer. Mol Carcinog 2007;46:503-511.

21 Abe N, Watanabe T, Suzuki Y, et al. An increased highmobility group A2 expression level is associated with malignant phenotype in pancreatic exocrine tissue. $\mathrm{Br}$ J Cancer 2003;89:2104-2109.

22 Miyazawa J, Mitoro A, Kawashiri S, et al. Expression of mesenchyme-specific gene HMGA2 in squamous cell 
carcinomas of the oral cavity. Cancer Res 2004;64: 2024-2029.

23 Rogalla P, Drechsler K, Kazmierczak B, et al. Expression of HMGI-C, a member of the high mobility group protein family, in a subset of breast cancers: relationship to histologic grade. Mol Carcinog 1997;19: 153-156.

24 Aliano S, Cirmena G, Garuti A, et al. HMGA2 overexpression in polycythemia vera with $\mathrm{t}(12 ; 21)$ (q14;q22). Cancer Genet Cytogenet 2007;177: 115-119.

25 Etienne A, Carbuccia N, Adelaide J, et al. Rearrangements involving $12 \mathrm{q}$ in myeloproliferative disorders: possible role of HMGA2 and SOCS2 genes. Cancer Genet Cytogenet 2007;176:80-88.

26 Storlazzi CT, Albano F, Locunsolo C, et al. $\mathrm{t}(3 ; 12)(\mathrm{q} 26 ; \mathrm{q} 14)$ in polycythemia vera is associated with upregulation of the HMGA2 gene. Leukemia 2006; 20:2190-2192.

27 Yunis AA, Arimura GK, Russin DJ. Human pancreatic carcinoma (MIA PaCa-2) in continuous culture: sensitivity to asparaginase. Int J Cancer 1977;19:128-135.

28 Jaffee EM, Schutte M, Gossett J, et al. Development and characterization of a cytokine-secreting pancreatic adenocarcinoma vaccine from primary tumors for use in clinical trials. Cancer J 1998;4:194-203.
29 Berman DM, Karhadkar SS, Maitra A, et al. Widespread requirement for Hedgehog ligand stimulation in growth of digestive tract tumours. Nature 2003;425:846-851.

$30 \mathrm{Xu} \mathrm{Y,} \mathrm{Sumter} \mathrm{TF,} \mathrm{Bhattacharya} \mathrm{R,} \mathrm{et} \mathrm{al.} \mathrm{The} \mathrm{HMG-I}$ oncogene causes highly penetrant, aggressive lymphoid malignancy in transgenic mice and is overexpressed in human leukemia. Cancer Res 2004;64: 3371-3375.

31 Di Cello F, Hillion J, Hristov A, et al. HMGA2 participates in transformation in human lung cancer. Mol Cancer Res 2008;6:743-750.

32 Maitra A, Fukushima N, Takaori K, et al. Precursors to invasive pancreatic cancer. Adv Anat Pathol 2005; 12:81-91.

33 Hezel AF, Kimmelman AC, Stanger BZ, et al. Genetics and biology of pancreatic ductal adenocarcinoma. Genes Dev 2006;20:1218-1249.

34 Talar-Wojnarowska R, Malecka-Panas E. Molecular pathogenesis of pancreatic adenocarcinoma: potential clinical implications. Med Sci Monit 2006;12 RA186-RA193.

35 Thuault S, Valcourt U, Petersen M, et al. Transforming growth factor-beta employs HMGA2 to elicit epithelial-mesenchymal transition. J Cell Biol 2006;174: 175-183. 